\title{
La existencia de escuelas privadas no elitistas $(*)$
}

\author{
Francisco Martínez Mora \\ University of Leicester, Reino Unido
}

\section{Resumen}

Este artículo ofrece una explicación del aparente contrasentido que supone la existencia de escuelas privadas de pago que ofrecen una calidad educativa inferior a la de algunas alternativas públicas de matrícula gratuita. Para ello se considera un modelo de una ciudad formada por dos comunidades: el área urbana y los suburbios. El colegio público de los suburbios proporciona servicios de educación de mayor calidad a un precio implícito determinado por una carga impositiva superior y un premio en el precio de la vivienda con respecto al del área urbana. Si ese precio implícito es suficientemente elevado y el colegio público del área urbana tiene un nivel de calidad suficientemente reducido, algunas familias de ingreso intermedio optan por vivir en el área urbana y utilizar un colegio privado no elitista. Tales colegios, privados y de calidad intermedia, aparecen para satisfacer la demanda de estas familias. Otras familias de renta tanto superior como inferior al de aquellas utilizan colegios públicos de diferente calidad. Por consiguiente, este equilibrio no se caracteriza por la estratificación perfecta de la población en función de la renta entre la educación pública y privada.

Palabras clave: Elección de comunidad, Elección de escuela, Estratificación, Jerarquía de calidades de escuela.

Clasificación JEL: H42; H73; I20; R13; R31

\begin{abstract}
We provide an explanation to the puzzle of the existence of paid-for private schools that offer lower quality education than some tuition-free public alternatives. We consider a model of a city composed of two communities: the urban area and the suburbs. The suburban public school provides higher quality education at an implicit price: the higher tax burden plus a housing rent premium. If that price is high enough and the urban public school has a sufficiently low quality, intermediate income households live in the urban area and use a private school. Intermediate quality private schools, then, exist to serve these households' demand. Lower and higher income households use different quality public schools. Therefore, perfect income stratification across public and private education does not characterize this equilibrium.
\end{abstract}

Keywords: Community choice; School choice; Stratification; Hierarchy of school qualities JEL classification: H42; H73; I20; R13; R31

* Esta es una versión adaptada de otro artículo publicado en el Journal of Public Economics. Agradezco a la editorial Elsevier por permitirnos esta adaptación [18]. Estoy profundamente agradecido a Gianni de Fraja por su orientación y comentarios y por discusiones siempre muy útiles. También tengo que agradecer los comentarios de Sam Bucovetsky, Charles de Bartolome, María Cubel, Elena del Rey, Manuel Martín Rodríguez, Diego Martínez, Fabien Moizeau, Thomas Nechyba, Socorro Puy, Javier Rodero, Daniel Santín, Albert Solé, y especialmente de mi director de tesis Rogelio Velasco, así como de Richard Romano. Los errores que pudieran subsistir son entera responsabilidad mía. Asimismo agradezco el apoyo financiero de la Junta de Andalucía, el Centro de Estudios Andaluces y FUNCAS. 


\section{Introducción}

Los colegios privados no siempre proporcionan una educación de calidad superior a la de los colegios públicos. Tanto la experiencia cotidiana como la evidencia empírica (Figlio y Stone, 1999) así lo confirman. Este hecho, sin embargo, choca frontalmente con el conocimiento convencional, la teoría básica de precios y la mayor parte de la literatura teórica previa sobre competición entre colegios públicos y privados (e.g., Epple y Romano, 1998). La cuestión es: ¿por qué pagaría una familia un colegio privado cuando puede obtener educación pública de matrícula gratuita y superior calidad?

La literatura sobre Economía de la Educación propone algunas posibles explicaciones. Figlio y Stone (1999) sostienen, de manera informal, que los colegios privados aparentemente ofrecen algunas ventajas no estrictamente académicas con respecto a los colegios públicos (por ejemplo, más disciplina y seguridad, un abanico más amplio de actividades extraescolares o, para algunos padres, una educación de carácter religioso). En esta misma línea, Brunello y Rocco (2005) demuestran de manera teórica que es posible que un colegio privado de pago de inferior calidad que la alternativa pública entre con éxito en el mercado estableciendo un nivel de exigencia inferior y ofreciendo en consecuencia más ocio a los estudiantes.

En este artículo se ofrece una explicación alternativa que no se apoya en diferencias no académicas entre colegios públicos y privados. Se basa, en cambio, en las interacciones entre la educación pública local, la tributación y el mercado de vivienda. Se construye un modelo de equilibrio general con dos comunidades (que para fijar ideas pueden denominarse el área urbana y los suburbios o la periferia), en el que coexisten colegios públicos locales y colegios privados competitivos. Se utiliza una versión computacional del modelo para construir ejemplos en los que pueden existir equilibrios múltiples. Estos equilibrios serán de dos tipos que vamos a denominar equilibrio de trampa urbana («urban-trap») y equilibrio de mezcla urbana («urban mixing»).

En un equilibrio de trampa urbana, las familias de renta baja viven en el área urbana y utilizan el colegio público local, mientras que los hogares de renta alta viven en los suburbios y envían a sus hijos al colegio público suburbano de mayor calidad. Existe además un conjunto de familias de renta intermedia que viven en el área urbana pero que no utilizan la escuela pública local porque demandan una educación de mayor calidad. Los colegios privados de calidad intermedia (no elitistas) aparecen para satisfacer esta demanda de educación. Por último, las familias más ricas pueden elegir entre adquirir educación privada de élite o vivir en los suburbios y utilizar el colegio público suburbano. Una economía puede encontrarse también en un equilibrio de mezcla urbana en el que, por el contrario, únicamente las familias con un nivel de renta superior a un cierto umbral adquieren educación privada (en este caso de élite). Las familias con un nivel de renta inferior a dicho umbral se estratifican perfectamente en función de la renta entre el área urbana y los suburbios, y envían a sus hijos al colegio público local correspondiente.

La intuición que se encuentra detrás de estos resultados es la siguiente. Los colegios públicos locales siguen una política de admisión que exige a los alumnos re- 
sidir en su área de influencia. Esto convierte a la vivienda y la educación pública (el derecho a asistir a un determinado colegio público) en bienes complementarios, dando lugar a un premio en el precio de la vivienda allí donde la educación pública es mejor (en nuestros ejemplos, en la periferia). Como consecuencia, el colegio de matrícula gratuita de los suburbios tiene un precio implícito suma del premio en el precio de la vivienda y de la mayor carga fiscal necesaria para financiar una educación de mayor calidad. En un equilibrio de trampa urbana, el colegio público del centro urbano ofrece una educación de muy baja calidad. Al mismo tiempo, las familias más ricas viven en la periferia, lo que aumenta tanto la calidad del colegio público de allí como su precio implícito. Como consecuencia, algunas familias de renta intermedia residen en el área urbana, más barata, pero puesto que valoran la educación, prefieren adquirir educación privada de calidad intermedia antes que enviar a sus hijos al colegio público de baja calidad.

Los casos en que se da un equilibrio de trampa urbana constituyen contraejemplos de un resultado muy sólido en la literatura previa. Los modelos de Stiglitz (1974), Epple y Romano (1996) y De Fraja (2001) predicen que, si las familias difieren en una sola dimensión, bien en renta o bien en la capacidad del estudiante, en equilibrio deberían estar perfectamente estratificadas entre la educación pública y la privada en función de la dimensión relevante; y las familias en mejor situación deberían utilizar colegios privados de mayor calidad. Esta predicción no se cumple en un equilibrio de trampa urbana: en tanto que las familias de renta intermedia eligen colegios privados, otros hogares tanto de renta inferior como superior prefieren utilizar una alternativa pública.

Los patrones de estratificación que caracterizan este tipo de equilibrio son comunes en muchas áreas metropolitanas estadounidenses. Al tiempo que la ciudad central ofrece una educación pública de baja calidad, la vivienda en las comunidades de la periferia resulta muy cara. En este contexto, es frecuente encontrar familias de renta relativamente baja que viven en la ciudad central y utilizan colegios privados de calidad intermedia (no elitistas). Es decir, como se argumenta en el artículo, la evidencia en Neal (1997), Figlio y Stone (1999) y en Altonji et al. (2005) está de acuerdo con las predicciones de un equilibrio de trampa urbana.

El resto del artículo se organiza como sigue: la Sección 2 presenta un modelo de dos comunidades que se inspira fundamentalmente en Epple y Romano $(1996,2002)$ y en Nechyba (1999). La Sección 3 define los equilibrios de trampa urbana y de mezcla urbana. En la Sección 4 se exponen los ejemplos computacionales de equilibrio y se presenta una discusión de los resultados obtenidos. Por último, en la Sección 5 se realizan algunas observaciones a modo de conclusión.

\section{El modelo}

Se estudia un modelo estático de equilibrio general sobre elección de comunidad y escuela. El modelo representa una ciudad formada por dos comunidades $C_{j}, j=u, s$, que se denominan respectivamente el área urbana y los suburbios o la periferia. La 
ciudad está habitada por un continuo de hogares o familias cuya masa se normaliza a uno. Todos los hogares se componen de un adulto y de un niño en edad escolar, y poseen preferencias idénticas definidas sobre la calidad de la educación que recibe el niño $(x)$ y el bien numerario de consumo $(b)$. Pensando en los ejemplos computacionales de equilibrio, se adopta la siguiente función de utilidad estrictamente cóncava, usada por Bearse et al. (2001):

$$
u(b, x)=\frac{1}{1-\sigma}\left[b^{1-\sigma}+\delta x^{1-\sigma}\right] ; \quad \sigma, \delta>0
$$

Cada familia pertenece a una de $I>2$ clases de renta o ingreso para $i=1, \ldots, I$, con $y_{i-1}<y_{i}, i=2, \ldots, I$. La proporción de población perteneciente al tramo de renta i se denota con $\lambda_{i}$. Las comunidades disponen de un stock fijo de viviendas homogéneas; cada hogar alquila una unidad de vivienda en la comunidad en la que reside al precio $p_{h}^{j}{ }^{1}$. La educación se representa como un bien privado que puede o no ser provisto por el sector público. Los colegios públicos y privados utilizan la misma tecnología para producir servicios educativos a partir del bien numerario. Las unidades de calidad se normalizan de modo que cada una cuesta una unidad del bien numerario por alumno ${ }^{2}$.

Cada comunidad impone un impuesto proporcional sobre el valor de la vivienda y utiliza la recaudación para proveer educación pública de calidad $E$. El par $(E, t)$, donde $t$ es el tipo impositivo, se elige a través de un proceso político, que se simplifica al de votación por mayoría. No existen transferencias del gobierno central: por tanto, en la comunidad $j$, la restricción presupuestaria del gobierno local es:

$$
E_{j}=t_{j} p_{h}^{j} \frac{N_{j}}{n_{j}} ; \quad j=u, s
$$

donde $N_{j}$ representa la proporción de familias que residen en la comunidad, y $n_{j}$ la proporción de aquellas que utilizan el colegio público local; el resto hace uso de colegios privados.

Los colegios públicos siguen políticas de admisión basadas en el lugar de residencia: si una familia quiere enviar a su hijo a un colegio público local determinado, tiene que vivir y pagar impuestos en la comunidad a la que pertenece. Las familias tienen además la posibilidad de renunciar a la opción pública y adquirir educación privada de cualquier nivel de calidad en un mercado competitivo.

Las decisiones se adoptan en dos etapas: en la primera, las familias eligen simultáneamente entre comunidades y colegios, teniendo en cuenta sus expecta-

1 Como es habitual en esta literatura, para cerrar el modelo se supone que un propietario ausente recibe todas las rentas de las viviendas.

2 Por simplicidad, esta tecnología ignora la influencia del efecto de grupo de pares («peer group effect») y otros inputs tales como el esfuerzo del estudiante. 
tivas sobre el vector de políticas públicas y precios de vivienda de equilibrio $e^{*}=\left(E_{u}, t_{u} p_{h}{ }^{u}, E_{s}, t_{s}, p_{h}{ }^{s}\right)$. En esta etapa, los mercados locales de vivienda se vacían. En la segunda etapa, una vez comprometida la elección de escuela y comunidad, las familias votan sobre la política educativa de su comunidad ${ }^{3}$.

En esta economía, un equilibrio consiste en una partición de hogares entre comunidades y colegios, una asignación $(\mathrm{b}, \mathrm{x})$ para cada hogar y un vector de políticas locales y precios de la vivienda $e^{*}$ que satisfacen las siguientes propiedades: (i) Elecciones racionales: cada familia maximiza su utilidad; (ii) equilibrio en el mercado de vivienda: la demanda de vivienda es igual a la oferta de vivienda en cada comunidad; (iii) equilibrio en la votación por mayoría: para $\mathrm{j}=\mathrm{u}, \mathrm{s}$, el par $\left(\mathrm{E}_{\mathrm{j}}, \mathrm{t}_{\mathrm{j}}\right)$ satisface la restricción presupuestaria del gobierno local y es preferida mayoritariamente por los votantes de la comunidad j, dada la partición de hogares entre colegios y el precio de la vivienda en la comunidad. Un par $\left(\mathrm{E}_{\mathrm{j}}, \mathrm{t}_{\mathrm{j}}\right)$ es preferido mayoritariamente en la comunidad $\mathrm{j}$ si es preferido por al menos la mitad del electorado de esa comunidad sobre cualquier otra alternativa que satisfaga la restricción presupuestaria del gobierno local en una votación con dos alternativas.

Las nociones de estratificación que adoptamos son las siguientes: decimos que un equilibrio muestra estratificación perfecta por ingreso entre la educación pública y la privada si toda familia que utiliza un colegio privado tiene un nivel de ingreso débilmente superior al de todas las familias que envían a sus hijos a un colegio público. Por otra parte, decimos que un equilibro muestra estratificación perfecta por ingreso a través de las comunidades si toda familia que reside en la comunidad rica tiene un nivel de renta débilmente superior al de todas las familias que habitan en la otra comunidad. Diremos además que una comunidad es mixta cuando algunos de sus habitantes utilizan el colegio público local mientras otros optan por una alternativa privada.

Este tipo de modelo ha sido ampliamente analizado en la literatura previa. Varios resultados son relevantes para el análisis que a continuación se desarrolla y se extienden fácilmente al marco analítico expuesto ${ }^{4}$ : (i) dentro de una comunidad mixta cualquier equilibrio muestra estratificación perfecta por ingreso entre la educación pública y la privada (Epple y Romano, 1996); (ii) si las comunidades difieren en la calidad de la educación pública en equilibrio, el precio de la vivienda incluido el impuesto es mayor en la comunidad con la mejor escuela pública ${ }^{5}$; (iii) las familias que eligen una escuela pública se estratifican perfectamente por renta entre las comunidades; de en-

3 Esta secuencia de decisiones también se encuentra en Nechyba (1999). Una secuencia alternativa permitiría a las familias elegir colegio después de la votación (Epple y Romano, 1996, Bearse et al., 2001). Sin embargo, esto puede dar lugar a problemas de existencia de equilibrio, dado que las preferencias no son unimodales en ese caso (Stiglitz, 1974).

${ }_{4}$ En la versión en documento de trabajo de este artículo (Martínez-Mora, 2003) se demuestra formalmente que todos estos resultados se extienden a este modelo. Allí se utiliza una función de utilidad genérica estrictamente cuasi-cóncava y no separable.

5 Esto se debe al requisito de residencia de los colegios públicos que hace que la vivienda y la educación pública se conviertan en bienes complementarios (Epple y Romano, 2002). 
tre ellas, las que tienen un nivel de ingreso mayor viven en la comunidad que ofrece una educación pública mejor ${ }^{6}$; (iv) las familias que adquieren educación privada residen en la comunidad en que el precio de la vivienda bruto de impuestos es menor ${ }^{7}$; (v) en cada comunidad existe un equilibrio en la votación por mayoría ${ }^{8}$.

\section{Equilibrio de trampa urbana vs. equilibrio de mezcla urbana}

En los ejemplos computacionales de equilibrio que se presentan más adelante, ambas comunidades ofrecen educación pública de diferente calidad, al tiempo que existen escuelas privadas. El colegio público del área urbana ofrece una calidad educativa inferior, lo que implica que el precio de la vivienda bruto de impuestos es menor allí. En este caso, el equilibrio puede ser de dos tipos que denominamos de trampa urbana («urban-trap») o de mezcla urbana («urban-mixing»).

Definición 1. Un equilibrio de trampa urbana es un equilibrio en el cual: (i) los hogares de renta baja viven en el área urbana y utilizan el colegio público de baja calidad; (ii) un grupo de familias de renta intermedia vive en el área urbana y adquiere educación privada de calidad intermedia; (iii) un conjunto de familias con un nivel de ingreso superior vive en los suburbios y envía a sus hijos al colegio público de calidad alta; y (iv) los hogares más ricos pueden bien vivir en el área urbana y adquirir educación privada de élite, o bien vivir en la periferia y utilizar el colegio público local ${ }^{9}$.

Definición 2. Un equilibrio de mezcla urbana es un equilibrio en el cual: (i) los hogares de renta baja viven en el área urbana y utilizan el colegio público local; (ii) los hogares de renta intermedia viven en los suburbios y utilizan el colegio público

6 Este resultado se debe a que el incremento en la disposición a pagar por una vivienda en respuesta a un aumento de la calidad de la escuela pública local crece monótonamente con la renta (véase, por ejemplo, Bénabou, 1996).

La vivienda y la educación no son bienes complementarios para estas familias, ya que no existe un vínculo entre la elección de comunidad y de colegio privado. Así, eligen vivir donde su renta neta (la renta tras pagar los impuestos y la vivienda) es máxima (Bearse et al., 2001; Nechyba, 1999).

8 Bajo la secuencia de decisiones del modelo, las preferencias sobre el tipo impositivo son unimodales en la etapa en que la votación tiene lugar. Se puede por tanto aplicar el teorema del votante mediano (Black, 1948). Hay que señalar que el equilibrio en la votación en una comunidad mixta mostrará la propiedad denominada «extremos contra el medio» («ends against the middle») investigada por Epple y Romano (1996). En consecuencia, el votante mediano tendrá un nivel de renta inferior a la renta mediana de la comunidad.

9 Bénabou (1993), Ranzagas (1995) y Epple y Romano (2002) han encontrado equilibrios similares a este. Epple y Romano analizan un modelo con una única jurisdicción dividida en múltiples barrios, cada uno con un colegio público. El modelo excluye al sector educativo privado. Bajo una política de admisión abierta, si existe un coste de transporte positivo, el equilibrio muestra estratificación residencial perfecta entre los barrios y algunas familias de ingreso intermedio que viven en el barrio pobre están dispuestas a pagar el coste de transporte con el fin de que su hijo reciba una educación de mayor calidad. Rangazas presenta un ejemplo de un equilibrio con estratificación completa en el que diferencias exógenas entre comunidades llevan a una estratificación perfecta por renta entre ellas. En este equilibrio, las familias más ricas en cada comunidad optan por la educación privada. En Bénabou (1993), la comunidad pobre puede no ser capaz de ofrecer educación de una calidad suficiente. En ese caso, su población entera puede optar por no realizar ningún esfuerzo educativo y unirse a la economía informal. 
de mayor calidad; (iii) las familias más ricas viven en el área urbana y adquieren educación privada de élite.

Las Figuras 1, 2 y 3 muestran la distribución de hogares entre comunidades y colegios en ambos tipos de equilibrio. Representan la relación entre la calidad de la educación recibida por cada estudiante y el nivel de renta de su familia, teniendo en cuenta las elecciones sobre comunidad y tipo de colegio de cada familia.

El equilibrio de trampa urbana contiene la principal contribución de este artículo. Los ejemplos de este tipo de equilibrio sirven para demostrar dos resultados novedosos sobre competición entre colegios públicos y privados: en un equilibrio con dos comunidades, (i) la distribución de hogares entre colegios públicos y privados no exhibe necesariamente estratificación perfecta por renta; y (ii) es posible (Figuras 1 y 2) que existan colegios privados de pago no elitistas (es decir, colegios privados que ofrecen una educación de calidad inferior a la alternativa pública de mayor calidad). Conviene destacar además que un equilibrio de trampa urbana muestra estratificación perfecta por renta entre comunidades en los casos en que las familias más ricas viven en los suburbios. En ese caso, la educación privada existe sin causar ninguna mezcla de hogares de diferentes tramos de renta en la ciudad central (Fig. 2).

Por su parte, un equilibrio de mezcla urbana muestra estratificación perfecta en función de la renta entre los sectores educativos público y privado. Esto es debido a que en este tipo de equilibrio únicamente las familias más ricas optan por adquirir edu-

FIGURA 1

EQUILIBRIO DE TRAMPA URBANA

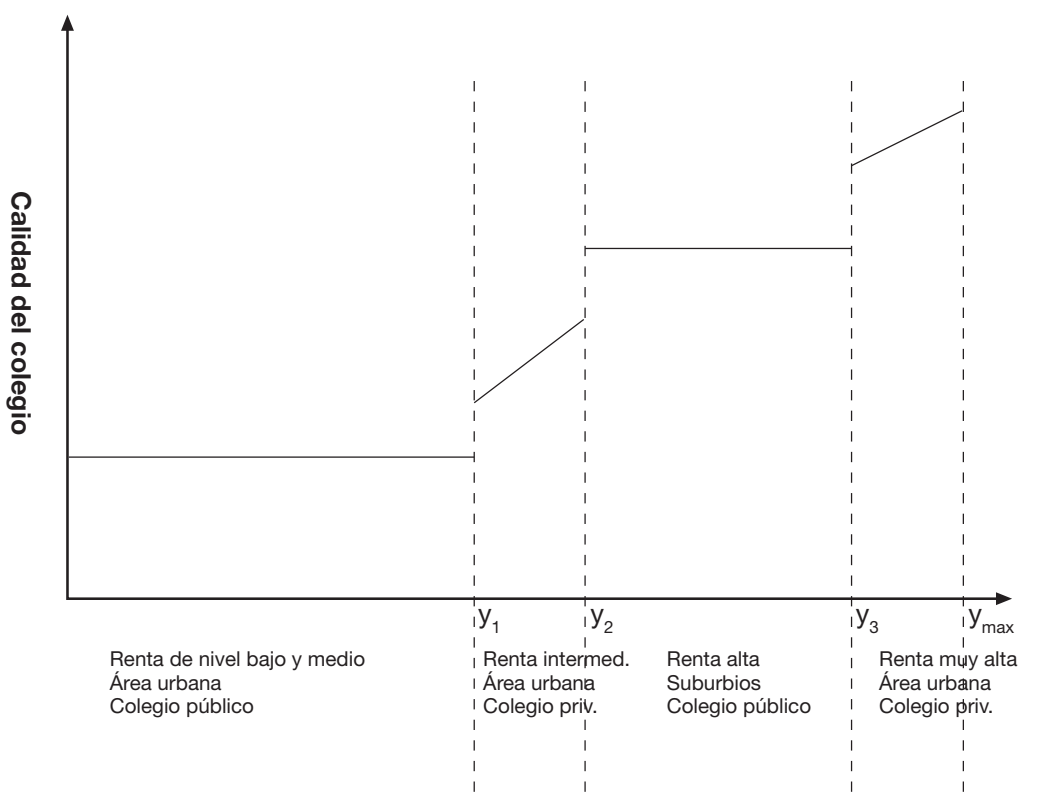

Renta 


\section{FIGURA 2}

\section{EQUILIBRIO DE TRAMPA URBANA}

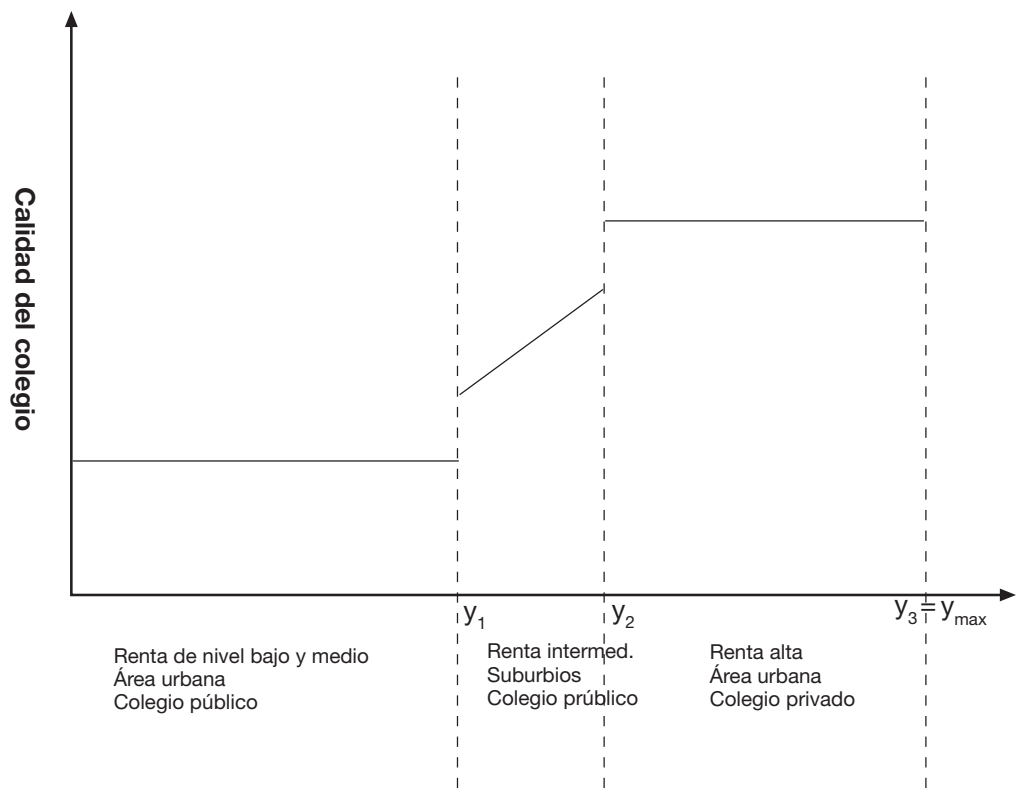

Renta

FIGURA 3

EQUILIBRIO DE MEZCLA URBANA

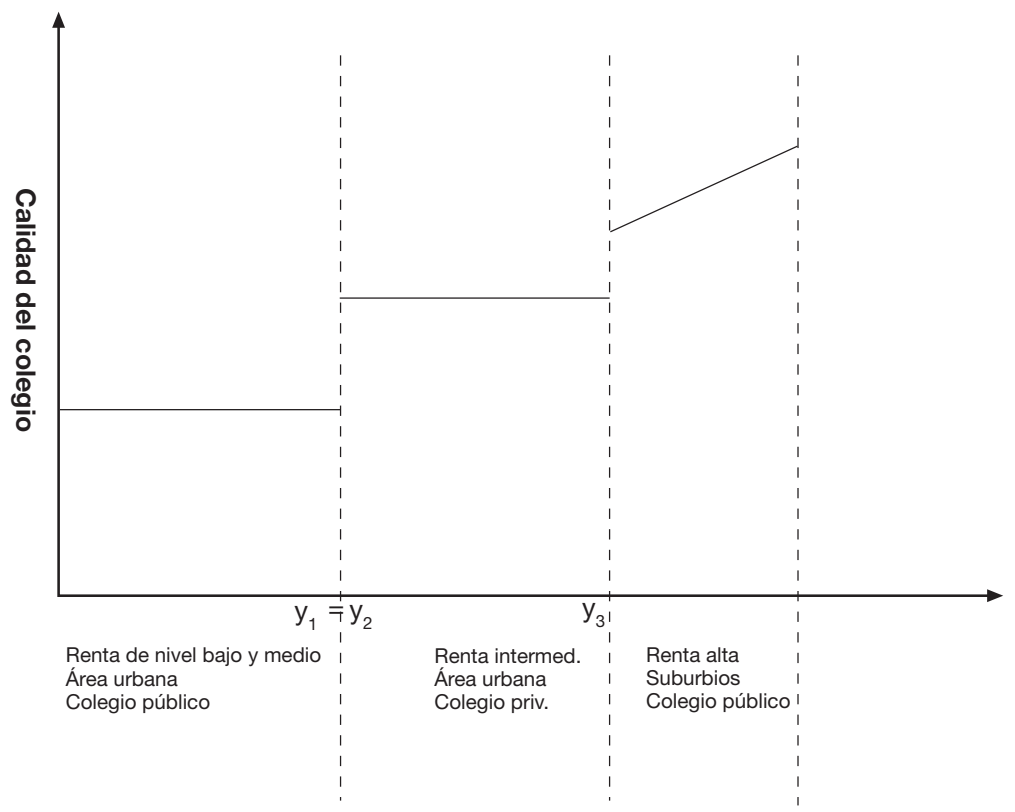

Renta 
cación privada (en este caso de élite). Estas familias viven en el área urbana, donde el precio de la vivienda incluido el impuesto es menor. Allí se mezclan con familias de renta baja que por el contrario mandan a sus hijos al colegio público local (Fig. 3). Las propiedades de lo que hemos denominado equilibrio de mezcla urbana ya han sido analizadas en la literatura previa (Bearse et al., 2001).

\section{Ejemplos computacionales y discusión}

A continuación, se presentan y discuten los ejemplos computacionales de equilibrio ${ }^{10}$. Con el fin de explicar la intuición e implicaciones del análisis con mayor claridad, se eligen dos economías para las que ambos tipos de equilibrio existen ${ }^{11}$. Veamos las características de estas economías: las tres primeras filas de la Tabla 1 contienen el valor de los parámetros. Cada distribución de renta es una versión discreta y truncada de una distribución continua de tipo lognormal. Ambas tienen veinte clases de renta que van desde $y_{\min }=5$ a $y_{\max }=100$ en intervalos constantes. La proporción de hogares en la clase de renta $i$-ésima viene dada por ${ }^{12}: \lambda_{i}=\Phi \cdot\left(F\left(y_{i}\right)-F\left(y_{i-1}\right)\right)$, $\forall i \neq 1$ con $\lambda_{1}=\Phi \cdot F\left(y_{1}\right)$. La función de utilidad viene dada por la ecuación (1) con los valores de $\sigma$ y $\delta$ mostrados en la tabla. En estos ejemplos, el área urbana es grande respecto a la periferia: $H_{u}$ es igual a 0,83 en el ejemplo 1 y a 0,91 en el ejemplo 2 . Finalmente, el coste de construcción de una casa se fija en $c_{h}=2,5$, lo que garantiza que todas las familias en la economía pueden al menos pagar el precio de la vivienda bruto de impuestos en el área urbana.

El resto de la Tabla 1 contiene el valor de variables endógenas clave. Presenta, para cada equilibrio, la calidad de los colegios públicos y privados, la renta del votante mediano, los tipos impositivos de equilibrio, el precio implícito del colegio público de la periferia $(\tau)$ y el porcentaje de estudiantes que acuden a colegios privados. Además, $y_{1}, y_{2}, y_{3}$ caracterizan la distribución de hogares entre comunidades y colegios: los hogares con un nivel de renta en el intervalo $y \in\left[y_{\min }, y_{l}\right]$ viven en el área urbana y utilizan el colegio público urbano; los hogares con niveles de renta $y \in\left[y_{1}, y_{2}\right]$ viven en el área urbana pero envían a sus hijos a una escuela privada; aquellos con niveles de renta $y \in\left[y_{2}, y_{3}\right]$ viven en los suburbios y utilizan el colegio público de allí; por último, los hogares con niveles de renta $y \in\left[y_{3}, y_{\max }\right]$ viven en el área urbana y utilizan un colegio privado de élite ${ }^{13}$.

10 Los cálculos que demuestran que los ejemplos son en verdad equilibrios del modelo están disponibles en www.le.ac.uk/ec/fmm14.

${ }^{11}$ Por supuesto, no cualquier economía presenta múltiples equilibrios.

$12 F$ es la función de densidad acumulada de la distribución lognormal, $\Phi$ es una constante de normalización que garantiza que $\Sigma_{i} \lambda_{i}$.

13 En un equilibrio de mezcla urbana, $y_{1}=y_{2}$ por lo que ningún hogar de renta intermedia opta por la educación privada. Por otro lado, si $y_{3}=y_{\max }$ en un equilibrio de trampa urbana, los hogares con mayor renta no optan por la educación privada y el equilibrio se caracteriza por la estratificación perfecta de la población entre comunidades en función de la renta. 


\section{TABLA 1}

\section{EJEMPLOS COMPUTACIONALES DE EQUILIBRIO}

\begin{tabular}{|c|c|c|c|c|}
\hline Parámetros & \multicolumn{2}{|c|}{ Ejemplo 1} & \multicolumn{2}{|c|}{ Ejemplo 2} \\
\hline Distribución de la renta & \multicolumn{2}{|c|}{$\begin{array}{c}y_{\text {mean }}=40,91 ; y_{\text {median }}=35 \\
y_{\text {min }}=5 ; y_{\text {max }}=100\end{array}$} & \multicolumn{2}{|c|}{$\begin{array}{c}y_{\text {mean }}=41,72 ; y_{\text {median }}=35 \\
y_{\text {min }}=5 ; y_{\text {max }}=100\end{array}$} \\
\hline \multirow[t]{2}{*}{$\begin{array}{l}\text { Función de utilidad } \\
\text { Mercado de la vivienda }\end{array}$} & \multicolumn{2}{|c|}{$\begin{array}{c}\sigma=1,83 ; d=0,0028 \\
c_{h}=2,50 ; H_{u}=0,83 ; H_{s}=0,17\end{array}$} & \multicolumn{2}{|c|}{$\begin{array}{c}\sigma=1,71 ; \delta=0,0026 \\
c_{h}=2,50 ; H_{u}=0,91 ; H_{s}=0,09\end{array}$} \\
\hline & $\begin{array}{c}\text { Trampa urbana } \\
\text { (1a) }\end{array}$ & $\begin{array}{l}\text { Mezcla urbana } \\
\text { (1b) }\end{array}$ & $\begin{array}{c}\text { Trampa urbana } \\
\text { (2a) }\end{array}$ & $\begin{array}{l}\text { Mezcla urbana } \\
\text { (2b) }\end{array}$ \\
\hline \multicolumn{5}{|c|}{ Políiticas públicas } \\
\hline $\begin{array}{l}E_{u} \\
E_{s} \\
t_{u} \\
t_{s} \\
\text { Renta del votante mediano, área urbana } \\
\text { Renta del votante mediano, suburbios }\end{array}$ & $\begin{array}{c}0,95 \\
2,97 \\
0,33 \\
0,93 \\
25 \\
80\end{array}$ & $\begin{array}{l}1,11 \\
2,58 \\
0,41 \\
0,76 \\
30 \\
70\end{array}$ & $\begin{array}{l}0,75 \\
2,59 \\
0,25 \\
0,84 \\
25 \\
90\end{array}$ & $\begin{array}{c}0,88 \\
1,99 \\
0,31 \\
0,61 \\
30 \\
70\end{array}$ \\
\hline \multicolumn{5}{|c|}{ Educación privada } \\
\hline $\begin{array}{l}\text { Calidad mínima de la educación privada } \\
\text { Calidad máxima de la educación privada } \\
\text { Estudiantes, colegios privados no-elitistas } \\
\text { Estudiantes, colegios privados }\end{array}$ & $\begin{array}{r}2,00 \\
3,74 \\
10,09 \\
11,67\end{array}$ & $\begin{array}{c}3,35 \\
3,73 \\
- \\
5,34\end{array}$ & $\begin{array}{r}1,70 \\
2,30 \\
15,23 \\
15,23\end{array}$ & $\begin{array}{c}2,29 \\
2,89 \\
- \\
9,96\end{array}$ \\
\hline \multicolumn{5}{|c|}{ Precios de la vivienda } \\
\hline $\begin{array}{l}p_{h}^{u} \\
p_{h}^{s} \\
p_{u}=p^{u}{ }_{h}\left(1+t_{u}\right) \\
p_{s}=p_{h}^{s}\left(1+t_{s}\right) \\
\tau=p_{s}-p_{u}\end{array}$ & $\begin{array}{l}2,50 \\
3,21 \\
3,32 \\
6,18 \\
2,86\end{array}$ & $\begin{array}{l}2,50 \\
3,37 \\
3,53 \\
5,95 \\
2,42\end{array}$ & $\begin{array}{l}2,50 \\
3,09 \\
3,12 \\
5,69 \\
2,57\end{array}$ & $\begin{array}{l}2,50 \\
3,27 \\
3,28 \\
5,26 \\
1,98\end{array}$ \\
\hline \multicolumn{5}{|c|}{ Rentas frontera } \\
\hline $\begin{array}{l}y_{1} \\
y_{2} \\
y_{3}\end{array}$ & $\begin{array}{l}50 \\
65 \\
95\end{array}$ & $\begin{array}{l}60 \\
60 \\
85\end{array}$ & $\begin{array}{c}55 \\
80 \\
100\end{array}$ & $\begin{array}{l}65 \\
65 \\
75\end{array}$ \\
\hline
\end{tabular}

Definición de rentas frontera: $y_{1}$, máximo nivel de renta de los hogares que residen en el área urbana y utilizan el colegio público local; $y_{2}$, mínimo nivel de renta de los hogares que residen en los suburbios y utilizan el colegio público local; $y_{3}$, máximo nivel de renta de los hogares que residen en los suburbios y utilizan el colegio público local. 
La posibilidad de que algunas familias de renta intermedia opten por adquirir educación privada no elitista se debe a las interacciones entre la educación pública local, la imposición local y los mercados de vivienda por un lado, y a la convexidad de las preferencias por otro. La provisión local de educación pública da lugar a diferencias entre comunidades en cuanto a la presión impositiva y al gasto en educación y, en consecuencia, en cuanto a la calidad de los colegios públicos. Las políticas de admisión basadas en el lugar de residencia que practican los colegios públicos, además, provocan que la vivienda y la educación pública se conviertan en bienes complementarios, lo que en equilibrio resulta en un incremento del precio de la vivienda en la comunidad con el colegio público de mayor calidad ${ }^{14}$. Como consecuencia, el colegio público de mayor calidad (y matrícula gratuita) situado en los suburbios tiene un precio implícito que es igual a la suma del premio en el precio de la vivienda y los mayores impuestos que pagan los residentes de esa comunidad: $\tau=p_{h}{ }^{s}\left(1+t_{s}\right)-p_{h}{ }^{u}\left(1+t_{u}\right)$.

Debido a que las preferencias son convexas, ese precio implícito puede hacer que algunas familias de renta intermedia encuentren insatisfactorias las combinaciones de consumo del numerario y calidad educativa asociadas a las alternativas públicas. Esto se muestra en la Figura 4 que ilustra la elección óptima de los hogares con una renta $y=45,55,65$ en el equilibrio de trampa urbana del ejemplo 1 . Las alternativas entre las que estas familias pueden elegir son: (i) vivir en el área urbana y utilizar el colegio público local (el punto triangular), (ii) vivir en el área urbana y adquirir educación privada de la calidad que deseen pagar, (el círculo), (iii) vivir en la periferia y enviar a su hijo al colegio público de la zona, (el cuadrado) ${ }^{15}$. Como muestra el gráfico, las familias con una renta $y=55$ maximizan su utilidad eligiendo la segunda de estas alternativa. Tal elección les proporciona una combinación intermedia preferida de calidad educativa y de consumo del numerario. La Figura 4 muestra además la elección óptima de hogares con renta $y=45$, que prefieren vivir en el área urbana y utilizar el colegio público local, así como la de aquellos con renta $y=65$, que prefieren vivir en los suburbios y enviar a sus hijos al colegio público local.

Hay que destacar que, como revelan los ejemplos anteriores, ambos tipos de equilibrio pueden existir para la misma economía. El que ésta termine en uno u otro depende, por un lado, del grado de apoyo político a la educación pública en el área urbana y, por otro, del resultado de la competición por una vivienda de los suburbios (que a su vez depende del proceso político local y de cómo varía con el nivel de ingreso la disposición a pagar por una vivienda allí). Para explicar esto con mayor de-

14 Para que esto suceda la oferta de vivienda no puede ser perfectamente elástica. Aquí se asume una oferta fija de vivienda para simplificar. Sin embargo, este premio en el precio de la vivienda también aparece en equilibrio en modelos con múltiples comunidades en los que la oferta de vivienda es imperfectamente elástica (véase por ejemplo Epple et al., 1984). Además, la existencia de mercados de vivienda no es una condición sine qua non para la existencia de equilibrios de trampa urbana. Es sencillo encontrar este tipo de equilibrio en una versión del modelo con impuestos de capitación y sin mercados de vivienda.

15 Hay que recordar que todas las familias que renuncian al sector público prefieren estrictamente vivir en el área urbana. 


\section{FIGURA 4 \\ ELECCIONES ÓPTIMAS}

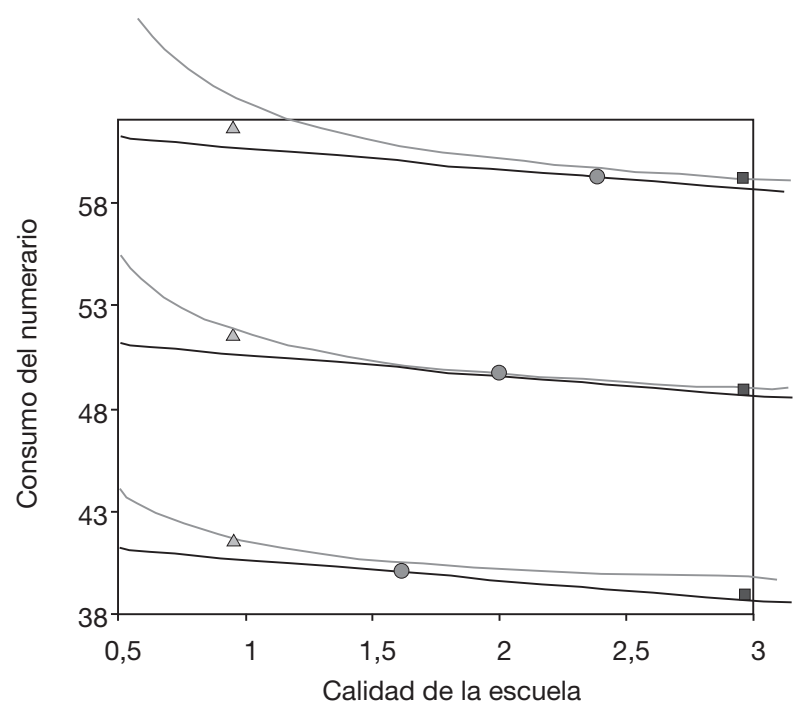

talle, definimos la función de puja $\hat{p}_{h}^{s}\left(y, E_{u}, t_{u}, p_{h}^{u}, E_{s}, t_{s}\right)$ que determina la máxima puja que una familia con un nivel de ingreso $y$ está dispuesta a ofrecer por una vivienda en los suburbios dados $E_{u}, t_{w}, p_{h}^{u}, E_{s} \mathrm{y} t_{s}$. Esta función de puja se escribe como sigue:

$$
\begin{array}{ll}
\hat{p}_{h}^{s}(\cdot)=\frac{y-\left[\left(y-p_{u}\right)^{1-\sigma}+\delta E_{u}^{1-\sigma}-\delta E_{s}^{1-\sigma}\right]^{\frac{1}{1-\sigma}}}{1+t_{s}} & \text { if } y \in\left[y_{\min }, y_{u}^{r}\right] ; \\
\hat{p}_{h}^{s}(\cdot)=\frac{y-\left[\left(y-p_{u}-x_{u}\left(y, p_{u}\right)\right)^{1-\sigma}+\delta x_{u}\left(y, p_{u}\right)^{1-\sigma}-\delta E_{s}^{1-\sigma}\right]^{\frac{1}{1-\sigma}}}{1+t_{s}} & \text { if } y \in\left[y_{u}^{r}, y_{\min }\right] .
\end{array}
$$

donde $p_{j}=p_{h}^{j}\left(1+t_{j}\right)$ es el precio de la vivienda bruto de impuestos en $C_{j}, x_{j}\left(y, p_{j}\right)$ es la función de demanda de educación privada, e $y_{u}^{r}$ es el nivel de renta para el cual una familia que reside en el área urbana es indiferente entre utilizar el colegio público local y enviar a su hijo a la alternativa privada preferida ${ }^{16}$. Los hogares con un nivel de renta $y$ tal que $\hat{p}_{h}^{s}\left((y, \cdot) \geq p_{h}{ }^{s}\right.$ viven en la periferia en equilibrio. A continuación, definimos $\hat{y}$ como el nivel de renta que iguala la demanda de educación privada que tendría

16 Desde Epple y Romano (1996) sabemos que todos los hogares con un nivel de ingreso por encima de este umbral () prefieren estrictamente una escuela privada y viceversa. Teniendo esto en cuenta, la función de puja se deduce fácilmente de la igualdad entre $u\left(y-p_{u}, E_{u}\right)=u\left(y-(\cdot)\left(1+t_{s}\right), E_{s}\right)$ en el intervalo de renta en el que $y<$, y de la igualdad entre $u\left(y-p_{u}-x_{u}\left(y, p_{u}\right), x_{u}\left(y, p_{u}\right)\right)=u\left(y-(\cdot)\left(1+t_{s}\right), E_{s}\right)$ para $y \geq$. Esta función es continua, ya que por definición $u\left(y-p_{u}-x_{u}\left(y, p_{u}\right), x_{u}\left(y, p_{u}\right)\right)=u\left(y-p_{u}, E_{u}\right)$ para $y=$, y diferenciable salvo para $y=$. 
una familia en caso de residir en $\mathrm{C}_{\mathrm{u}}$ a $E_{s}$, es decir tal que $x_{u}\left(\hat{y}, p_{u}\right)=E_{s}$. El Lema 1 analiza el comportamiento de $\hat{p}_{h}^{s}$ como una función del nivel de renta.

Lema 1. Para $E_{u}<E_{s}, \hat{p}_{h}^{s}(\cdot)$ es cuasi-cóncava en el nivel de renta, y alcanza su máximo global para $y=\hat{y}$ si $y_{u}^{r}<\hat{y}$, y para $y=y_{u}^{r}$ si $y_{u}^{r} \geq \hat{y}$.

Prueba. Dados $E_{u}, t_{u}, p_{h}^{s}, E_{s}$ y $t_{s}$, denotamos con $\rho_{a}(y)$ y $\rho_{b}(y)$ a la primera y segunda «piezas» de $\hat{p}_{h}^{s}(\cdot)$, respectivamente. Para $E_{u}<E_{s}$, la suma de la máxima puja que una familia con un nivel de renta inferior a $y_{u}^{r}$ estaría dispuesta a ofrecer por una vivienda en $C_{s}$ y del impuesto que la misma implicaría pagar, $\rho_{a}(y)\left(1+t_{s}\right)$, es siempre mayor que el precio de la vivienda incluido el impuesto en el área urbana, $p_{u}$. Esto hace que $\rho_{a}$ sea creciente en el nivel de ingreso:

$$
\rho_{a}^{\prime}(y)=\frac{\left[y-\rho_{a}(y)\left(1+t_{s}\right)\right]^{-\sigma}-\left(y-p_{u}\right)^{-\sigma}}{\left[y-\rho_{a}(y)\left(1+t_{s}\right)\right]^{-\sigma}\left(1+t_{s}\right)}>0
$$

La segunda pieza de $\hat{p}_{h}^{s}(\cdot), \rho_{b}(y)$, tiene un punto estacionario para $y=\stackrel{\circ}{y}$ tal que $\dot{y}-\rho_{b}(\dot{y})\left(1+t_{s}\right)=-p_{u}-x_{u}\left(\dot{y}, p_{u}\right)$, puesto que en ese punto:

$$
\rho_{b}\left(\begin{array}{c}
\dot{y} \\
)
\end{array}\right) \frac{\left[\dot{\circ}-\rho_{b}(\dot{y})\left(1+t_{s}\right)\right]^{-\sigma}-\left[\dot{o}-p_{u}-x_{u}\left(\dot{o}, p_{u}\right)\right]^{-\sigma}}{\left[\dot{0}-\rho_{b}(\dot{y})\left(1+t_{s}\right)\right]^{-\sigma}\left(1+t_{s}\right)}=0
$$

Después de alguna manipulación, la segunda derivada de $\rho_{b}$ para $y=\stackrel{0}{y}$ se puede escribir así:

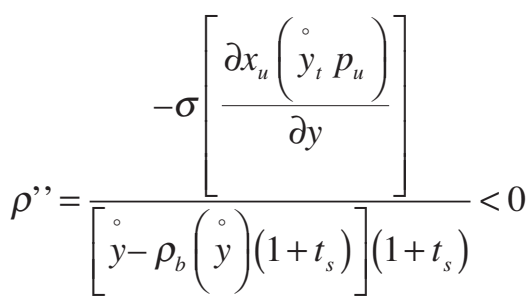

Esta expresión es siempre negativa porque la demanda de educación es normal. Esto implica que $\rho_{b}$ tiene un único punto estacionario, que es además su máximo global. Ahora consideremos $\hat{y}$. Por definición, $x_{u}\left(\hat{y}, p_{u}\right)=E_{s}$; en consecuencia $\rho_{b}(\hat{y})$ debe ser tal que el nivel de consumo del numerario (y por tanto de utilidad) de las familias con un ingreso igual a $\hat{y}$ se iguale entre las alternativas relevantes, esto es tal que: $\hat{y}$ $\rho_{b}(\hat{y})\left(1+t_{s}\right)=\hat{y}+p_{u}-x_{u}\left(\hat{y}, p_{u}\right)$. Esta es la condición para que $\rho_{b}(y)$ tenga un punto estacionario en $\hat{y}$. Por lo tanto, como $\rho_{a}\left(y_{u}{ }^{r}\right)=\rho_{b}\left(y_{u}{ }^{r}\right), \hat{p}_{u}{ }^{r}$ es cuasi-cóncava en el nivel de ingreso y tiene un único máximo para $y=\hat{y}$ si $y_{u}{ }^{r}<\hat{y}$, y para $y=y_{u}{ }^{r}$ en caso de que $y_{u}{ }^{r} \geq \hat{y}$. 
En virtud del Lema 1, la disposición a pagar por una vivienda en los suburbios aumenta con la renta en el tramo en que las familias prefieren el colegio público del área urbana al sector privado. Una vez que la renta supera el umbral $y_{u}{ }^{r}$, en cambio, la puja que un hogar está dispuesto a ofrecer es tanto mayor cuanto más cerca esté su demanda de calidad educativa de la ofrecida por el colegio público de los suburbios. Por este motivo, se incrementa con la renta para todo $y$ tal que $x_{u}\left(y, p_{u}\right)<E_{s}$, se reduce con la renta para todo $y$ tal que $x_{u}\left(y, p_{u}\right)>E_{s}$, y alcanza su máximo para $y=\hat{y}$.

Tal como se puede observar en la Tabla 1, en un equilibrio de trampa urbana el nivel de calidad del colegio público de la periferia es muy alto. Esto supone que las familias ricas, cuya demanda de calidad educativa está cerca de aquel nivel, estén dispuestas a pagar más por una vivienda en los suburbios que las familias con menor renta y con una demanda de calidad educativa inferior (Fig.5a). Al mismo tiempo, el colegio público del área urbana ofrece educación de calidad muy baja. En consecuencia, algunos hogares de renta intermedia que están «atrapados» en el área urbana, optan por renunciar al sector público y adquieren educación privada de calidad intermedia. La asignación de hogares a comunidades y sectores educativos que resulta sostiene el nivel de calidad de ambos colegios públicos como un equilibrio en la votación local correspondiente. En el área urbana una proporción elevada de familias optan por un colegio privado, de modo que el proceso político resulta en un nivel reducido de gasto por alumno y una calidad educativa también reducida. La población rica que reside en los suburbios, por su parte, apoya un gasto por alumno elevado y, por consiguiente, una educación pública de calidad.

Por el contrario, en un equilibrio de mezcla urbana las familias más ricas no están dispuestas a pagar tanto como algunos hogares con una renta inferior por una casa en los suburbios (Fig. 5b). El motivo es que en este caso la calidad educativa que demandan es mayor que la ofrecida por el colegio público local. Como consecuencia, viven en el área urbana y adquieren educación privada de mayor calidad. Las familias de renta intermedia, por su parte, no renuncian a la educación pública. Por un lado, algunas de ellas están dispuestas a pagar el precio necesario para vivir en los suburbios con el fin de acceder a su colegio público. Por otro lado, la calidad del colegio público del área urbana no es lo bastante baja como para inducir a las familias de renta intermedia que residen allí a renunciar a la educación pública.

FIGURA 5a

\section{EQUILIBRIO DE TRAMPA URBANA}

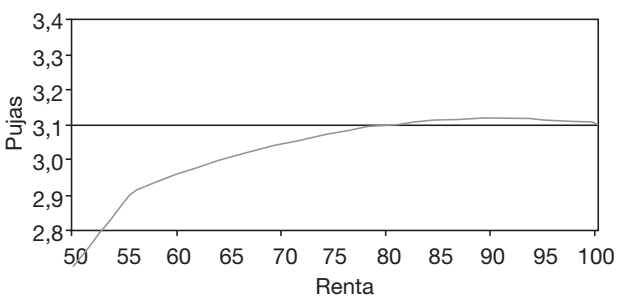

FIGURA 5b

\section{EQUILIBRIO DE MEZCLA URBANA}

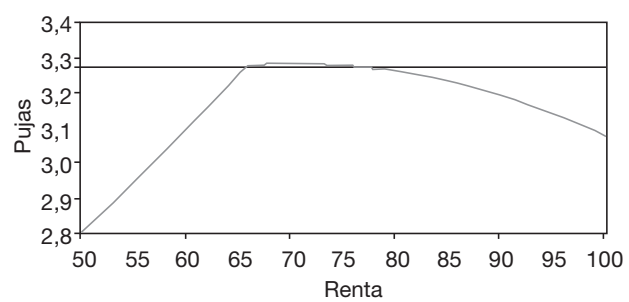


Merece la pena hacer algunas observaciones adicionales. En primer lugar, existe evidencia empírica para los EEUU que sugiere que los colegios privados (católicos) tienen un impacto positivo sobre el rendimiento de los estudiantes de áreas urbanas pertenecientes a minorías étnicas (e.g. Neal, 1997; Figlio y Stone, 1999; Altonji et al., 2005). Esta evidencia es consistente con los patrones de estratificación que caracterizan al equilibrio de trampa urbana. Esto no es sorprendente puesto que este tipo de equilibrio representa un cuadro que es frecuente en las áreas metropolitanas de los EEUU: algunos estudiantes del área urbana asisten y, especialmente aquellos pertenecientes a minorías étnicas, se benefician de los colegios privados católicos porque (en palabras de Neal): «sus alternativas educativas públicas son pobres y porque sus familias pueden permitirse el modesto gasto de matrícula comunes en los colegios católicos, pero raramente pueden permitirse una vivienda en los barrios exclusivos con los mejores colegios públicos». Esto ya fue apreciado por Friedman (1962) ${ }^{17}$.

En segundo lugar, el equilibrio de trampa urbana tiene propiedades claramente indeseables desde el punto de vista de la equidad. La calidad de enseñanza que reciben los estudiantes de familias de renta baja es menor. Esto se encuentra asociado a un mayor tamaño del sector educativo privado, que a su vez incrementa el nivel de segregación en función del ingreso en escuelas individuales. Además, el argumento que sugiere que la educación privada tiende a reducir la estratificación residencial por ingreso (e.g. Nechyba, 1999; Bearse et al., 2001) es más débil cuando se refiere a este tipo de equilibrio: como pone de manifiesto el ejemplo 2 , un equilibrio de trampa urbana puede mostrar estratificación perfecta por renta entre comunidades.

En tercer lugar, la posibilidad de que una economía esté en un equilibrio de trampa urbana debería considerarse en el análisis de las propuestas de bonos educativos («vouchers»). Nechyba $(1999,2000)$ y Luengo-Prado y Volij (2003) han investigado las consecuencias de diferentes políticas de bonos educativos en un marco con múltiples comunidades. Parece importante extender esta línea de investigación a una economía que se encuentre en este tipo de equilibrio antes de que se introduzcan los bonos educativos. En este sentido, Rangazas (1995) dio un primer paso al construir un ejemplo de su equilibrio con estratificación completa en el que los bonos educativos para escuelas privadas conducen a un aumento de la calidad de las escuelas públicas tanto en la comunidad rica como en la pobre.

Finalmente, nuestros resultados arrojan luz sobre el modo en que los colegios privados se distribuyen a lo largo del espacio de calidad. En particular, el equilibrio de trampa urbana explica que algunos colegios privados son muy buenos al tiempo que otros no lo son tanto (Marks et al., 2001), y apoya la idea de que los colegios privados aparecen para cubrir huecos dentro del menú de alternativas públicas.

17 Otra rama de investigación empírica se centra en los determinantes de las elecciones de los hogares entre colegios públicos y privados. Un resultado de esta literatura también parece apoyar la relevancia empírica del equilibrio de trampa urbana. Buddín et al. (1998) obtienen que, ceteris paribus, es más probable que los estudiantes de raza negra y los blancos hispanos asistan a un colegio privado. Una explicación plausible es que, ceteris paribus, es más probable que estos estudiantes vivan en áreas marginales de las ciudades, donde los colegios públicos son pobres. 


\section{Conclusiones}

El mercado educativo en una economía con múltiples comunidades es complejo. De acuerdo con el conocimiento convencional, cabría esperar que, en un equilibrio con colegios públicos y privados, los alumnos de familias ricas fueran a colegios privados y el resto utilizara la educación pública. Además, todos los colegios privados deberían ofrecer una calidad superior, ya que nadie pagaría por ellos si existiera una alternativa gratuita de mayor calidad. Este artículo explica que esto no tiene por qué suceder así en un contexto con múltiples comunidades. La educación pública de calidad tiene un precio implícito compuesto por una mayor carga impositiva y un premio en el precio de la vivienda. Como consecuencia, puede suceder que algunas familias de clase media no estén dispuestas a pagar el precio por vivir en una comunidad rica con una escuela pública buena. Si además la educación pública disponible en la comunidad en la que viven no es de suficiente calidad, estas familias adquirirán educación privada de calidad intermedia.

Este equilibrio, que hemos denominado de trampa urbana, refleja una situación frecuente en las áreas metropolitanas estadounidenses: el centro urbano ofrece educación pública de baja calidad, mientras que los suburbios resultan muy exclusivos. Esto hace que algunas familias de renta relativamente baja, que no pueden permitirse vivir en la periferia, adquieran educación privada no elitista. Un cuadro como éste parece estar detrás de la evidencia empírica que aportan Neal (1997), Figlio y Stone (1999) y Altonji et al. (2005). Esta rama de investigación empírica puede por tanto beneficiarse de nuestros resultados.

La posibilidad de que una economía se encuentre en un equilibrio de trampa urbana genera inquietud por sus implicaciones de equidad: los colegios públicos de las ciudades centrales (no las escuelas públicas de élite) podrían ser los más perjudicados por el efecto de la educación privada conocido como de descremado («cream-skimming»). Como resultado, los alumnos que se quedaran en estos colegios públicos tras la introducción de una política de bonos educativos podrían enfrentarse a obstáculos infranqueables para superar con éxito la etapa de educación obligatoria. Esto debería tenerse en cuenta a la hora de diseñar y analizar propuestas de bonos educativos.

\section{Referencias bibliográficas}

[1] ALTONJI, J.G., T.E. ELDER y C.R. TABER (2005): « Selection on observed and unobserved variables: assessing the effectiveness of Catholic schools», Journal of Political Economy, 113 (1), 151-184.

[2] BEARSE, P., G. GLOMM y B. RAVIKUMAR (2001): «Education Finance in a Dynamic Tiebout Economy».

[3] BÉNABOU, R. (1993): «Workings of a city: location, education and production», Quarterly Journal of Economics, 108 (3), 619-652. 
[4] BÉNABOU, R. (1996): «Equity and efficiency in human capital investment: the local connection», Review of Economic Studies, 63 (2), 237-264.

[5] BLACK, D. (1948): «On the rationale of group decision making», Journal of Political Economy, 56, 23-34.

[6] BRUNELLO, G. y L. ROCCO (2005): «Educational standards in private and public schools», CESIFO Working Paper, 1418.

[7] BUDDIN, R.J., J.J. CORDES y S.N. KIRBY (1998): «School choice in California: who chooses private schools?», Journal of Urban Economics, 44, 110-134.

[8] DE FRAJA, G. (2001): «Private and public schools: theoretical considerations», Lavoro e Relazioni Industriali, 2/2001, 49-82.

[9] EPPLE, D. y R. ROMANO (1996): «Ends against the middle: determining public service provision when there are private alternatives», Journal of Public Economics, 62, 297-325.

[10] EPPLE, D. y R. ROMANO (1998): «Competition between private and public schools, vouchers and peer group effects», American Economic Review, 88, 828-858.

[11] EPPLE, D. y R. ROMANO (2002): «Neighbourhood schools, choice and the distribution of educational benefits», en: Hoxby, C.M. (Ed.), The Economics of School Choice. National Bureau of Economic Research, University of Chicago Press, 227-286.

[12] EPPLE, D., R. FILIMON y T. ROMER (1984): «Equilibrium among local jurisdictions: toward an integrated treatment of voting and residential choice», Journal of Public Economics, 24, 281-308.

[13] FIGLIO, D y J. STONE (1999): «Are private schools really better?», in: Polachek, S.W. (Ed.), Research in Labor Economics, vol. 17, , 115-140. Amsterdam North-Holland

[14] FRIEDMAN, M. (1962): Capitalism and Freedom, The University of Chicago Press.

[15] LUENGO-PRADO, M.J. y O. VOLIJ (2003): «Public education, communities and vouchers», Quarterly Review of Economics and Finance, 43, 51-73.

[16] MARKS, J., J. BURN, P. PILKINGTON y P. THOMPSON (2001): Faith in Education, The Institute for the Study of Civil Society, London.

[17] MARTÍNEZ-MORA, F. (2003): «Opting-Out of Public Education in Urban Economies», Centro de Estudios Andaluces Working Paper, vol. E2003/52.

[18] MARTINEZ MORA, F. (2006): The existence of non-elite private schools, Journal of Public Economics, 90, 1505-1518

[19] NEAL, D. (1997): «The effect of catholic secondary schooling on educational attainment», Journal of Labor Economics, 15, 98-123.

[20] NECHYBA, T.J. (1999): «School finance induced migration patterns: the case of private school vouchers», Journal of Public Economic Theory, 1, 5-50.

[21] NECHYBA, T.J. (2000): «Mobility, targeting and private school vouchers», American Economic Review, 90, 130-146.

[22] RANGAZAS, P. (1995): «Vouchers in a community choice model with zoning. Quarterly Review of Economics and Finance», 35, 15-39.

[23] STIGLITZ, J.E. (1974): «The demand for education in public and private school systems», Journal of Public Economics, 3, 349-385. 
